\title{
Leucaena leucocephala (Lam.) de Wit (Fabaceae): INVASORA OU RUDERAL?¹
}

\author{
José Nicola Martorano Neves da Costa² e Giselda Durigan ${ }^{3}$
}

\begin{abstract}
RESUMO - (Leucaena leucocephala (Lam.) de Wit (Fabaceae): invasora ou ruderal?). A utilização de espécies exóticas em plantios de recuperação de áreas degradadas tem sido condenada com base no argumento de que tais espécies podem se comportar como invasoras e contaminar os ecossistemas naturais ao redor das áreas onde forem plantadas. Leucaena leucocephala (leucena) é espécie leguminosa exótica que tem sido frequentemente cultivada no Brasil para recuperação florestal, uma vez que apresenta simbiose com bactérias fixadoras de nitrogênio, melhorando a fertilidade dos solos. O potencial de invasão e persistência da leucena foi analisado a partir de um plantio misto, efetuado em 1983 em terreno com afloramento rochoso, parte do mosaico de uma paisagem dominada por matriz agrícola, com alguns fragmentos remanescentes de floresta estacional semidecidual e plantios arbóreos diversos. Em uma área de 200 ha, cada uma das 11 unidades do mosaico foi amostrada por meio de seis parcelas de 16 x $3 \mathrm{~m}\left(48 \mathrm{~m}^{2}\right)$, em que foram identificados e medidos (DAP) todos os indivíduos de espécies arbóreas (altura mínima de $50 \mathrm{~cm}$ ), para verificar se a área ocupada pela espécie está se expandindo. Na área em que foi utilizada leucena no plantio, analisou-se a estrutura da comunidade, com base na distribuição dos indivíduos em classes de tamanho, para verificar se a proporção da espécie exótica tende a aumentar com o tempo, configurando a persistência no ecossistema. Uma vez que nenhum indivíduo de $L$. leucocephala foi registrado entre os 4.599 amostrados além dos limites da área onde a espécie foi plantada, a conclusão é de que a espécie não está se comportando como invasora de ecossistemas naturais nessa condição ambiental, enquadrando-se melhor como ruderal. A análise da comunidade em regeneração sob as árvores plantadas evidenciou que a proporção da espécie exótica, que é intolerante à sombra, tende a diminuir com o tempo, ainda que lentamente, perdendo espaço para espécies nativas tolerantes, que poderão ocupar o dossel no futuro.
\end{abstract}

Palavras-chave: Leucena, Invasões biológica e Dinâmica de comunidades.

\section{Leucaena leucocephala (Lam.) de Wit (Fabaceae): INVASIVE OR RUDERAL?}

\begin{abstract}
Leucaena leucocephala (Lam.) de Wit (Fabaceae): invader or ruderal?) Exotic species have not been recommended for degraded lands reforestation, since they are reported to invade natural ecosystems in the surrounding areas. Leucena leucocephala, a leguminous $N$ fixing species, was introduced in Brazil and has been widely cultivated, especially to recover degraded soils. The potential of $\boldsymbol{L}$. leucocephala to expand over the landscape and its persistence in the plant community in the long term was analyzed. A stand planted in 1983 was assessed, which was installed on a rocky soil, immerse in an agricultural matrix, where some native and planted forest patches exists. Over a 200 ha area, eleven distinct patches were surveyed, where all individuals from arboreal species (minimum height $50 \mathrm{~cm}$ ) were identified and measured (dbh), to verify if the exotic species is expanding over areas where it has not been planted. In the stand where the species was planted, the community structure was assessed on the basis of relative density in size classes to verify if the proportion of the exotic species tends to increase with time, confirming persistence. Since, among the total of 4,599 individuals surveyed, not even a single individual of the species was recorded beyond the limits of the planted stand, invasion of natural ecosystems by Leucena leucocephala was refuted, the species being locally considered as ruderal. Even though a huge number of young individuals of $\mathbf{L}$. leucocephala are regenerating under the planted trees, the relative density of the exotic species in the understorey tends to decrease with time. Apparently, some shade tolerant native species can slowly dominate the community in the future, taking advantage under light competition.
\end{abstract}

Keywords: Leucena, Biological invasion e Community dynamics.

\footnotetext{
${ }^{1}$ Recebido em 28.11.2009 e aceito para publicação em 02.03.2010.

${ }^{2}$ Instituto Socioambiental - ISA, Canarana, MT, Brasil. E-mail: <jnicolamn@yahoo.com.br>.

${ }^{3}$ Instituto Florestal, Floresta Estadual de Assis -Assis SP, Brasil. E-mail: <giselda@femanet.com.br>.
} 


\section{INTRODUÇÃO}

O conceito de espécie invasora tem sido amplamente discutido na literatura (RICHARDSON et al., 2000; DAEHLER, 2001; DAVIS e THOMPSON, 2001; REJMÁNEK et al., 2002), sem que haja consenso. Regra geral é aceito que seja reputada como invasora uma espécie cuja população se expanda sobre ecossistemas nos quais não ocorre naturalmente, reduzindo a abundância ou deslocando espécies nativas, podendo, inclusive, alterar o funcionamento do ecossistema natural (SCHOFIELD, 1989; VITOUSEK, 1986). Tais espécies têm sido consideradas como uma das principais causas de perda de biodiversidade em todo o mundo (MILLER et al., 1989; WILCOVE, 1998; LEVINE et al., 2003; COLLAUTTI e MACISAAC, 2004), além de acarretarem sérios prejuízos econômicos (WILCOVE et al., 1998; PIMENTEL et al., 2000).

Para uma espécie não nativa que prolifera apenas em áreas perturbadas ou degradadas, porém, outras denominações têm sido utilizadas, como ruderal (WOITKE e DIETZ, 2002), termo adotado neste estudo. A diferença entre ambas as categorias é de alta relevância, quando se discute a necessidade de controle dos processos de invasão (HULME, 2006).

A leucena está entre as espécies leguminosas de rápido crescimento, fixadoras de nitrogênio, que têm despontado como alternativa promissora (FRANCO e FARIA, 1997; RESENDE e KONDO, 2001) para a recuperação da cobertura vegetal e reabilitação de áreas degradadas. Originária da América Central e amplamente distribuída, a leucena tem sido amplamente cultivada também devido à ampla gama de usos que se faz da espécie (FRANCO e SOUTO, 1986; LINS et al., 2007). Porém, há diversos estudos atribuindo à leucena a característica de planta invasora em diversas regiões do mundo (SMITH, 1985; WAGNER et al., 1999; SCHERER et al., 2005), que levaram à sua inclusão na lista das 100 espécies invasoras mais agressivas do planeta, elaborada pela União Mundial para a Conservação da Natureza - IUCN (LOWE et al., 2000).

Há relatos de que a espécie pode formar maciços densos, excluindo outras plantas e que, se não for controlada, pode avançar rapidamente sobre áreas adjacentes (SMITH, 1985; INSTITUTO HORUS, 2008). Todavia, há muito poucos estudos científicos voltados para a elucidação do processo de invasão pela leucena e não existem evidências científicas de que a espécie prolifere em ecossistemas naturais. Além disso, alguns ambientes são mais propícios à invasão e a invasibilidade de uma espécie pode ser variável entre ambientes e regiões (REJMÁNEK, 1999), devendo ser tratada como fenômeno biogeográfico e não taxonômico (COLLAUTTI e MACISAAC, 2004).

Leucaena leucocephala reúne alguns atributos típicos de espécies com alto potencial como invasoras, que são árvores de crescimento rápido (BLOSSEY e NÖTZOLD, 1995), pioneiras heliófitas (REJMÁNEK, 1996) e produzem sementes em grande quantidade (NOBLE, 1989). A espécie reúne, ainda, vários atributos que são considerados favoráveis para ervas daninhas invasoras (BAKER et al., 1965, 1974), quais sejam: capacidade de se reproduzir sexual e assexuadamente (leucena não se multiplica vegetativamente, mas rebrota sucessivas vezes após o corte), crescimento rápido, curto período pré-reprodutivo, alta plasticidade e tolerância a ambientes diversos.

Ainda que suas sementes secas e duras sejam predominantemente dispersas por gravidade (barocoria), há relatos de que a espécie é zoocórica (INSTITUTO HORUS, 2008) e que suas sementes podem ser dispersas por aves e formigas, possibilitando transporte para além do limite de suas copas, o que é requisito para a disseminação de espécies invasoras. A dispersão pelo homem (antropocoria), através de cultivo, tem sido uma das formas mais eficazes de disseminação de plantas invasoras (THOMPSON, 2007) e a leucena tem sido amplamente cultivada no Brasil, especialmente como planta forrageira e, também, com a finalidade de recuperação ambiental.

Caso esteja, de fato, comportando-se como invasora, a utilização de Leucaena leucocephala não seria recomendável em plantios visando à recuperação de ecossistemas ou à reabilitação de áreas degradadas e, em caso de dano comprovado aos ecossistemas naturais, sua erradicação poderia ser recomendada em regiões fitoecológicas semelhantes.

Este estudo foi realizado com os objetivos de verificar se: 1) em região de Floresta Estacional Semidecidual com matriz agrícola a leucena, plantada em área restrita com a finalidade de reabilitação, comporta-se como espécie invasora, expandindo-se por outras unidades do mosaico da paisagem; e 2) se, na área em que foi plantada, a espécie tende a dominar a comunidade em detrimento das espécies nativas, o 
que configuraria persistência em longo prazo. Dentro do segundo objetivo, testou a hipótese nula de que a abundância relativa da espécie tende a se manter estável no tempo, mediante duas hipóteses alternativas: a) a proporção de plantas de leucena em regeneração é maior nas classes de maior tamanho (vantagem competitiva sobre espécies nativas, tendência de dominar a comunidade com o tempo); e b) a proporção de plantas de leucena é menor nas classes de maior tamanho (desvantagem competitiva e tendência a perder espaço para as espécies nativas com o tempo).

\section{MATERIAL E MÉTODOS}

Este estudo faz parte de um projeto mais amplo, em que foram analisadas todas as unidades da paisagem de uma propriedade rural agrícola, para compreensão da contribuição de diferentes peças do mosaico na conservação e recuperação da biodiversidade (resultados em COSTA, 2008). O plantio de leucena é uma das peças desse mosaico e foi objeto das análises aqui apresentadas, para as quais foram utilizados os dados gerados pelo projeto como um todo.

\subsection{Local de estudo}

Aárea analisada corresponde aos limites da Fazenda Canaçu, situada na região do Médio Vale do Paranapanema (coordenadas aproximadas $22^{\circ} 45^{\prime}$ 'S e 50 25’W), Sudoeste do Estado de São Paulo, no Município de Tarumã. O clima regional é do tipo Cfa, de Köppen (1948), ou seja, mesotérmico, com temperatura dos meses mais frios inferiores a $18{ }^{\circ} \mathrm{C}$ e dos meses mais quentes superiores a $22{ }^{\circ} \mathrm{C}$, sujeito a geadas esporádicas e com estação seca no inverno. O solo é do tipo Latossolo Vermelho eutroférrico, segundo o Sistema Brasileiro de Classificação de Solos (EMBRAPA, 1999), e a precipitação média anual é de 1.350 mm (SILVEIRA e DURIGAN, 2004).

Com base na classificação de Veloso et al. (1991), a vegetação regional anterior à ocupação agrícola era Floresta Estacional Semidecidual. A cobertura de vegetação natural no Município de Tarumã encontra-se reduzida a apenas 2\% (KRONKA et al., 2005), em consequência do avanço da agricultura na região, que possui solo de elevada fertilidade. Atualmente, a paisagem do município é formada por matriz de agricultura intensiva, em que predomina a cana-de-açúcar, seguida de grãos (soja e milho) e mandioca.

A paisagem da Fazenda Canaçu é formada por fragmentos de floresta nativa, áreas em regeneração natural, pomares e plantios de restauração, imersos na matriz agrícola (Tabela 1). O plantio de Leucaena leucocephala foi efetuado em 1983, ocupando área de 0,63 ha, em um pequeno trecho, onde há afloramento rochoso, cerca de 200 m distante da zona ripária e a $200 \mathrm{~m}$ do fragmento florestal mais próximo. Efetuou-se semeadura direta, em linhas, com 3 m de distância entre linhas. Em parte da área do talhão e nas falhas do plantio de leucena, foram plantadas espécies florestais nativas, por mudas, a saber: Anadenanthera macrocarpa (Benth.) Brenan, Cariniana estrellensis (Raddi) Kuntze, Pterogyne nitens Tul., Tabebuia heptaphylla (Vell.) Toledo e Tabebuia impetiginosa (Mart. ex A.DC.) Standl.

Tabela 1 - Unidades do mosaico da paisagem ao redor do plantio de reabilitação com Leucaena leucocephala, que foram objeto de amostragem de espécies arbóreas (Município de Tarumã, Estado de São Paulo). Dist.: distância, em linha reta, do plantio de leucena. Ano: ano de plantio, quando área de restauração.

Table 1 - Landscape units around rehabilitation planting using Leucaena leucocephala subjected to arboreal species assessment (Tarumã municipality, state of São Paulo, Brazil). Dist.: distance from the rehabilitation planting. Ano: planting year, if restoration area.

\begin{tabular}{|c|c|c|c|}
\hline Unidade & Área (ha) & Dist. (m) & Ano \\
\hline Plantio misto de reabilitação com leucena & 0,63 & $\%$ & 1988 \\
\hline Fragmento de vegetação nativa 1 & 3,06 & 200 & - \\
\hline Fragmento de vegetação nativa 2 & 4,24 & 550 & - \\
\hline Fragmento de vegetação nativa 3 & 4,61 & 1100 & - \\
\hline Pomar & 2,67 & 1350 & 1973 \\
\hline Plantio heterogêneo de espécies nativas para restauração de mata ciliar & 0,60 & 200 & 1990 \\
\hline Plantio de espécies nativas em linhas (50\% pioneiras) para restauração da mata ciliar & 0,60 & 200 & 1990 \\
\hline Plantio puro de Pinus em zona ripária & 0,60 & 200 & 1990 \\
\hline Plantio puro de aroeira em zona ripária & 0,85 & 1600 & 1986 \\
\hline Pastagem abandonada com espécies arbóreas em regeneração & 15,97 & 1450 & 1968 \\
\hline Mata ciliar em regeneração natural sob pomar abandonado & 1,20 & 1200 & 1958 \\
\hline
\end{tabular}




\subsection{Método de amostragem}

Seis parcelas foram sistematicamente distribuídas dentro de cada unidade do mosaico, sendo a distância entre parcelas variável conforme a área ocupada pela unidade. Cada parcela ocupava área de 3 x 16 m (48 m²), em que foram medidas (diâmetro à altura do peito-DAP) e identificadas todas as árvores plantadas (leucena e espécies nativas) e todos os indivíduos de espécies arbóreas em regeneração, com altura mínima de 50 cm. Efetuou-se a identificação das espécies em campo ou, posteriormente, por comparação com exsicatas da flora regional depositadas na Coleção Botânica da Floresta Estadual de Assis. As espécies em regeneração sob o plantio de leucena foram classificadas quanto à síndrome de dispersão com base na literatura (YAMAMOTO et al., 2007). No caso da leucena, a dispersão usual é barocórica, mas em áreas com pastoreio as sementes são dispersas pelo gado (STAPLES et al., 2000) e há menção de que formigas podem atuar como dispersores secundários da espécie (SEIFFERT e THIAGO, 1983).

\subsection{Análise dos dados}

Para quantificar a possível invasão de leucena em outros ambientes além da área em que foi plantada, foram analisados os dados coletados em todas as unidades do mosaico da paisagem. No talhão de leucena, caracterizou-se a estrutura da comunidade, separando as árvores plantadas (área basal e densidade) das plantas em regeneração.

Para o estrato regenerante, efetuou-se a análise da distribuição dos indivíduos (de leucena e de espécies nativas) em classes de tamanho, separando-os em duas classes: indivíduos com DAP inferior a $1 \mathrm{~cm}$ e indivíduos com DAP igual ou superior a $1 \mathrm{~cm}$.

Para verificar se a proporção de indivíduos de leucena na comunidade está se modificando com o tempo, foi aplicado o teste chi-quadrado à frequência de ocorrência de indivíduos dessa espécie e de espécies nativas nas duas classes de tamanho, partindo do pressuposto de que os indivíduos de maior tamanho, para uma mesma espécie, têm idade superior. Para o teste chi-quadrado, considerou-se que o valor esperado seria de proporções iguais para as diferentes classes de tamanho, que comprovaria a hipótese nula (a proporção da espécie na comunidade tende a se manter estável com o tempo).

\section{RESULTADOS}

Nas 11 unidades do mosaico vegetacional na área de estudo, incluindo a área com leucena, foram amostrados 5.571 indivíduos, pertencentes a 138 espécies lenhosas. Do total, 209 eram indivíduos adultos plantados, e 5.362 correspondiam a indivíduos não plantados, incluindo as árvores adultas dos fragmentos e os regenerantes, de espécies nativas ou exóticas (COSTA, 2008). Nenhum indivíduo de Leucaena leucocephala foi amostrado além dos limites da área onde a espécie foi plantada.

A comunidade vegetal no talhão com leucena apresentou densidade de 1.527 árvores plantadas por hectare, das quais $45 \%$ são da espécie exótica, sendo os demais indivíduos distribuídos entre as espécies nativas. O estrato regenerante (a partir de $50 \mathrm{~cm}$ de altura) apresentou densidade total de 32.222 indivíduos por hectare, dos quais 93\% são de Leucaena leucocephala, os demais pertencendo a 20 espécies (Tabela 2), das quais apenas duas estão entre as que foram plantadas.

A densidade relativa de Leucaena leucocephala entre os regenerantes foi de $94 \%$ na classe de menor tamanho (DAP $<1 \mathrm{~cm}$ ) e $82 \%$ na classe de maior tamanho. A análise da diferença na proporção de indivíduos de leucena nas diferentes classes de tamanho dos regenerantes (Figura 1$)\left(\chi_{0,001,1 \mathrm{~g} .1}^{2}=17,89\right)$ confirmou que a densidade relativa da espécie exótica é menor entre os indivíduos maiores.

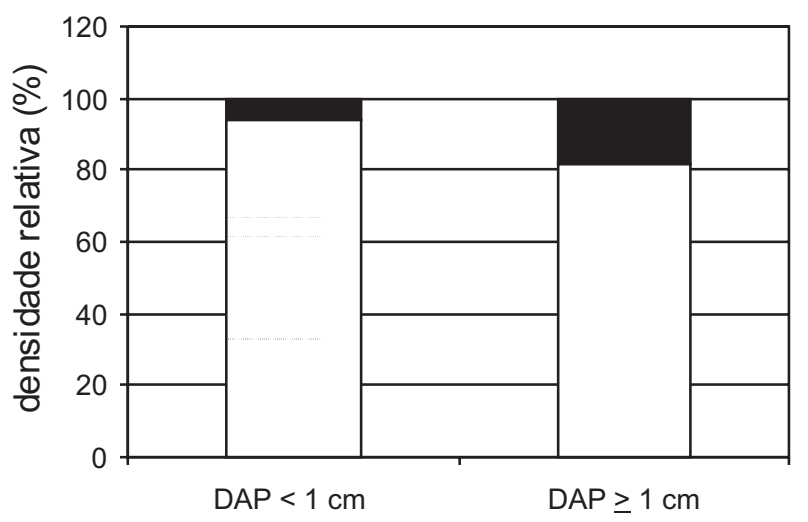

$\square$ Leucena leucocephala $\square$ outras espécies

Figura 1 - Densidade relativa, em duas classes de tamanho, de Leucaena leucocephala e de outras espécies arbóreas em regeneração sob plantio de reabilitação ambiental (Município de Tarumã, SP), em duas classes de tamanho.

Figure 1 - Relative density of Leucaena leucocephala and other arboreal species regenerating under rehabilitation planting (Tarumã municipality, state of São Paulo, Brazil), in two size classes. 
Tabela 2 - Espécies arbóreas, síndrome de dispersão e número de indivíduos em regeneração em duas classes de tamanho, sob plantio de reabilitação com Leucaena leucocephala (Município de Tarumã, Estado de São Paulo). N: número de indivíduos amostrados; e DAP: diâmetro à altura do peito (1,30 m).

Table 2 - Arboreal species, dispersal syndrome and number of individuals from two size classes, growing under the rehabilitation stand with Leucaena leucocephala (Tarumã municipality, state of São Paulo, Brazil). N: number of individuals sampled. DAP $($ dbh = diameter at breast height, $1.30 \mathrm{~m})$.

\begin{tabular}{|c|c|c|c|}
\hline Espécie & Síndrome de dispersão & $\mathrm{N}(\mathrm{DAP}<1 \mathrm{~cm})$ & $\mathrm{N}(\mathrm{DAP} \geq 1 \mathrm{~cm})$ \\
\hline Allophylus edulis (A.St.-Hil.) Radlk. & Zoocórica & 1 & \\
\hline Aloysia virgata (Ruiz \& Pav.) Juss. & Anemocórica & & 1 \\
\hline Anadenanthera macrocarpa (Benth.) Brenan & Autocórica & 2 & \\
\hline Cestrum strigilatum Ruiz \& Pav. & Zoocórica & 1 & \\
\hline Chrysopyllum gonocarpum (Mart. \& Eichler) Engl. & Zoocórica & 3 & \\
\hline Citrus sp. & Zoocórica & 3 & \\
\hline Cordia ecalyculata Vell. & Zoocórica & 1 & \\
\hline Genipa americana L. & Zoocórica & 4 & \\
\hline Guarea kunthiana A.Juss. & Zoocórica & 1 & \\
\hline Leucena leucocephala (Lam.) de Wit & Autocórica, Zoocórica & 788 & 72 \\
\hline Machaerium stipitatum (DC.) Vogel & Anemocórica & 8 & 10 \\
\hline Murraya paniculata (L.) Jacq. & Zoocórica & 6 & \\
\hline Nectandra megapotamica (Spreng.) Mez & Zoocórica & 2 & \\
\hline Ocotea puberula (Rich.) Nees & Zoocórica & 1 & \\
\hline Solanum mauritianum Scop. & Zoocórica & 2 & \\
\hline Solanum pseudoquina A.St.-Hil. & Zoocórica & 1 & \\
\hline Tabebuia chrysotricha (Mart. ex A.DC.) Standl. & Anemocórica & 1 & 4 \\
\hline Tabebuia impetiginosa (Mart. ex A.DC.) Standl. & Anemocórica & 1 & \\
\hline Tabernaemontana catharinensis A.DC. & Zoocórica & 7 & \\
\hline Trema micrantha (L.) Blume & Zoocórica & 1 & \\
\hline Trichilia pallida Swartz & Zoocórica & 6 & 1 \\
\hline TOTAL & & 840 & 88 \\
\hline
\end{tabular}

\section{DISCUSSÃO}

Para que seja, de fato, reputada como invasora, é necessário que, sem intervenção humana, uma espécie prolifere em ecossistemas naturais, reduzindo a abundância ou deslocando espécies nativas (RICHARDSON et al., 2000). Com base nesse conceito, no caso analisado neste estudo a leucena não está se comportando como espécie invasora, uma vez que não está se expandindo sobre ecossistemas naturais e nem mesmo ocupando novas áreas além da área de cultivo. A espécie seria, portanto, mais bem classificada como ruderal, uma vez que pode proliferar em áreas perturbadas e dificultar o estabelecimento de espécies nativas. Mediante a classificação proposta por Williamson e Fitter (1996), Leucaena leucocephala, nas condições da área estudada, pode ser considerada estabelecida, mas não oferece ameaça aos ecossistemas naturais e nem prejuízos econômicos.

Pouco mais de duas décadas após o plantio, a população de Leucaena leucocephala encontra-se estabelecida e com abundante regeneração na área onde foi plantada. No entanto, a diminuição da densidade relativa da espécie na comunidade com o tempo (há relativamente poucos indivíduos na classe de maior tamanho) sugere que sua persistência no sistema pode não se consolidar no longo prazo. Analisando o comportamento de espécies exóticas reputadas como invasoras em ambiente florestal no Rio de Janeiro, Siqueira (2006) incluiu a Leucena leucocephala entre as espécies que, embora constem das listas de espécies invasoras, não estão se comportando como tal. Conforme observado por Rejmánek (1999) e por Colautti e MacIsaac (2004), alguns ambientes são mais propícios à invasão, e a invasibilidade de uma espécie pode ser variável entre ambientes e entre regiões.

Ainda que reúna alguns predicados favoráveis à invasão (porte arbóreo, crescimento rápido e grande produção de sementes), as árvores de leucena não atingem grande porte (máximo de 5 a $7 \mathrm{~m}$ de altura, segundo Lorenzi et al. 2003), que é um dos atributos que caracterizam as plantas invasoras mais bemsucedidas (PRITCHARD, 1960; BLOSSEY e NÖTZOLD,

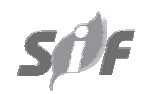

Revista Árvore, Viçosa-MG, v.34, n.5, p.825-833, 2010 
1995). Além disso, em sendo uma espécie pioneira heliófita, pode levar desvantagem se espécies nativas de maior porte conseguirem se estabelecer e sombrear os adultos reprodutivos. Em experimento visando determinar os mecanismos que conferem resistência de comunidades vegetais à invasão, Green et al. (2004) constataram 100\% de mortalidade das plântulas de Leucaena leucocephala no interior da floresta intacta. Esses autores consideraram a baixa luminosidade como o mais importante filtro abiótico impedindo a invasão por essa espécie.

A facilidade de regeneração à sombra tem sido considerada como indicativo do potencial invasivo de espécies arbóreas (e.g. Tabebuia rosea (Bertoloni) A.P.DC., por Socolowsky e Takaki 2007). A importância da luz para o estabelecimento da leucena em áreas antropizadas foi confirmada também por Colon e Lugo (2006). Abaixa luminosidade poderia explicar a inexistência de regenerantes de leucena nas unidades do mosaico ocupadas por florestas (nativas ou plantadas) neste estudo. Porém, limitações de dispersão parecem ser mais importantes localmente, uma vez que não foi registrada a ocorrência de leucena nem mesmo nas unidades com baixa cobertura arbórea (pomar e pastagem abandonada). A área de agricultura que separa o talhão de leucena dos outros ambientes analisados com, no mínimo, 200 m de distância parece ser intransponível para agentes da fauna que possam dispersar as sementes dessa espécie. Entre as 20 espécies amostradas em regeneração, 16 são zoocóricas, dispersas por aves ou morcegos, capazes de transpor aquela distância. A dispersão das sementes de leucena na área de estudo parece ser exclusivamente autocórica. Assim, as frequentes operações de preparo de solo para cultivo ao redor do talhão impedem que as plântulas de leucena se estabeleçam, evitando o aumento da área ocupada pela espécie.

A disseminação de espécies exóticas tem sido um dos argumentos contrários ao restabelecimento de corredores conectando fragmentos florestais isolados (PANETTA e HOPKINS, 1991; PIENIMÄKI e LEPPÄKOSKI, 2004). Donald e Evans (2006) argumentaram que a matriz agrícola pode ser benéfica em um esquema agricultura-conservação (AES), ao dificultar a disseminação de espécies invasoras entre módulos de conservação dispersos em uma matriz agrícola. No caso deste estudo, isso parece ser verdadeiro. Caso a matriz fosse pastagem, por exemplo, a área ocupada pela leucena poderia estar se expandindo. A matriz ocupada alternadamente por culturas anuais (soja, milho, trigo) ou cana-de-açúcar, durante mais de duas décadas desde o plantio, tem sido capaz de impedir que a espécie exótica se dissemine pela paisagem sem, contudo, impedir que algumas espécies zoocóricas ou anemocóricas nativas cheguem e se estabeleçam sob as árvores plantadas de leucena.

A alta densidade de leucena neste e em outros plantios, todavia, dificulta o estabelecimento de espécies nativas, comportamento típico de espécies ruderais agressivas. Yoshida e Oka (2004) constataram que a recuperação da biodiversidade e da estrutura de florestas nativas é muito mais lenta em áreas ocupadas por Leucaena leucocephala do que em áreas não ocupadas por essa espécie. Por essa razão, o uso da espécie deve se restringir a situações em que o ambiente esteja de tal modo degradado que seu desempenho não possa ser superado por espécies da flora local e que recuperar serviços ambientais seja prioritário. Nessas condições, o plantio simultâneo de espécies nativas perenifólias de grande porte que se adaptem ao ambiente degradado, em consorciação com a leucena, pode ser testado como alternativa promissora. As árvores de leucena, nesse caso, forneceriam rapidamente proteção aos solos e melhoria de sua qualidade pela fixação de nitrogênio e, com o tempo, perderiam espaço na comunidade à medida que as árvores nativas sombreassem suas copas, dificultando a reprodução e regeneração da espécie exótica.

A utilização de Leucaena leucocephala não é recomendável quando a meta do plantio for a recuperação da diversidade de espécies arbóreas. No entanto, o caráter ruderal da espécie em regiões fitoecológicas semelhantes à deste estudo não justifica políticas de erradicação, que deve ser obrigatória apenas para espécies que efetivamente invadem ecossistemas naturais e deslocam espécies nativas ou trazem prejuízos econômicos relevantes. Com base nos resultados deste estudo, acredita-se que o manejo adequado possa reverter a dominância da leucena em plantios puros já existentes. É de se supor que o desbaste progressivo acelere o ingresso de espécies nativas na comunidade, caso existam fontes de sementes e agentes dispersores nas proximidades. Desbaste seguido de plantio de enriquecimento com espécies nativas deve ser experimentado na inexistência de fontes próximas de propágulos. 


\section{AGRADECIMENTOS}

Ao Conselho Nacional do Desenvolvimento Científico e Tecnológico - CNPq, pelas bolsas de mestrado e produtividade em pesquisa, respectivamente, ao primeiro e segundo autores; e a José Bendito Lourenço e Helaine Souza, pelo auxílio nos levantamentos de campo.

\section{REFERÊNCIAS}

Baker, H.G. 1965. Characteristics and modes of origin of weeds. Pp 147-168. In Baker, H.G. e Stebbins, G.L. (eds.) The genetics of colonizing species. New York: Academic Press.

Baker, H.G. 1974. The evolution of weeds. Annual Review of Ecology and Systematics 5:1-24.

Blossey, B. e Nötzold, R. 1995. Evolution and increased competitive ability in invasive nonindigenous plants: a hypothesis. Journal of Ecology 83:887-889.

Colautti, R.I. e MacIsaac, H.J. 2004. A neutral terminology to define 'invasive' species.

Diversity and Distributions 10:135-141.

Colon, S.M. e Lugo, A.E. 2006. Recovery of a subtropical dry forest after abandonment of different land uses. Biotropica 38(3):354-364.

Costa, J.N.M. 2008. Efeito de diferentes modelos de plantios de restauração e dos grupos funcionais das espécies plantadas na estrutura da comunidade e suas contribuições na riqueza e diversidade da paisagem agrícola. Dissertação de Mestrado em Ciências da Engenharia Ambiental. Escola de Engenharia de São Carlos, da Universidade de São Paulo. 100 p.

Daehler, C.C. 2001. Two ways to be an invader, but one is more suitable for ecology. Bulletin of the Ecological Society of America 82:101-102.

Davis, M.A. e Thompson, K. 2001. Invasion terminology: should ecologists define their terms differently than others? No, not if we want to be of any help! Bulletin on the Ecological Society of America 82:206.
Donald, P.F. e Evans, A.D. 2006. Habitat connectivity and matrix restoration: the wider implications of agri-environment schemes. Journal of Applied Ecology 43:209-218.

Empresa Brasileira de Pesquisa Agropecuária EMBRAPA. Centro Nacional de Pesquisa de Solos. 1999. Sistema Brasileiro de Classificação de Solos. Rio de Janeiro, 412 p.

Franco, A. A. e Souto, S. M. 1986. Leucaena leucocephala - uma leguminosa com múltiplas utilidades para os trópicos. Seropédica, RJ: EMBRAPA - UAPNPBS. (Comunicado técnico: n. 2).

Franco, A.A. e Faria, S.M. 1997. The contribution of N2 fixing tree legumes to land reclamation and sustainability in the tropics. Soil Biology and Biochemistry 29(5/6):897-903.

Green, P.T.; Lake, P.S.; O’Dowd, D.J. 2004. Resistance of Island Rainforest to Invasion by Alien Plants: Influence of Microhabitat and Herbivory on Seedling Performance. Biological Invasions 61:1-9.

Hodkinson, D.J. e Thompson, K. 1997. Plant dispersal: the role of man. Journal of Applied Ecology 34:1484-1496.

Hulme, P.E. 2006. Beyond control: wider implications for the management of biological invasions. Journal of Applied Ecology, n. 43. p. 835-847.

Instituto Horus de Desenvolvimento e Conservação Ambiental/The Nature Conservancy. Leucaena leucocephala. 2008. Disponível em: http:// www.institutohorus.org.br/download/fichas/ Leucaena_leucocephala.htm (Acesso em 29/06/2008).

Kronka, F. J. N. et al. 2005. Inventário Florestal da Vegetação Natural do Estado de São Paulo.

São Paulo: Secretaria do Meio Ambiente / Instituto Florestal/ Imprensa Oficial.

Levine, J.M.; Vilà, M.; D’Antonio, C.M.; Dukes, J.S.; Grigulis, K. \& Lavorel, S. 2003. Mechanisms underlying the impacts of invasive species. Proceedings of the Royal Society 270:775-781.

Revista Árvore, Viçosa-MG, v.34, n.5, p.825-833, 2010 
Lins, C.E.L. et al. 2007. Efeito de fungos micorrízicos arbusculares no crescimento de mudas de Leucaena leucocephala (Lam.) de Wit. em solos de caatinga sob impacto de mineração de cobre. Revista Árvore 31(2):355-363.

Lorenzi, H. et al. 2003. Árvores exóticas no Brasil: madeireiras, ornamentais e aromáticas. Nova Odessa: Instituto Plantarum.

Lowe, S. et al. 2000. 100 of the world's worst invasive alien species: a selection from the global invasive species database. The Invasive Species Specialist Group/Species Survival Commission/ World Conservation Union IUCN.

Miller, R.R.: Williams, J.D. e Williams, J.E. 1989. Extinctions of North American fishes during the past century. Fisheries 14(6):22-38.

Noble, I.R. 1989. Attributes of invaders and the invading process: terrestrial and vascular plants. Pp 301-313. In Drake, J.A.; DiCastri, F.; Groves, R.H.; Kruger, F.J.; Mooney, H.A.; Rejmánek, M. \& Williamson, M.H. (eds.) Biological Invasions: a global perspective. New York: Willey.

Panetta, F.D. e Hopkins, A.J.M. 1991. Weeds in corridors: invasion and management. Pp. 341-351. In Saunders, D.A. e Hobbs, R.J. (eds.) Nature Conservation 2: the role of Corridors. Chipping Norton: Surrey Beatty and Sons.

Pienimäki, M. e Leppäkoski, E. 2004. Invasion pressures on the Finnish Lake District: invasion corridors and barriers. Biological

Invasions 6:331-346.

Pimentel, D.; Lach, L.; Zuniga, R. e Morrison, D. 2000. Environmental and economic costs of nonindigenous species in United States.

BioScience 50:53-65.

Pritchard, T. 1960. Race formation in weedy species with special reference to Euphorbia cyparizzias L. and Hypericum perforatum L. Pp 61-66. In Harper, J.L. (ed.). The biology of weeds: a symposium of the British Ecological Society. Oxford, 2-4 April 1959. Oxford: Blackwell Science.

Revista Árvore, Viçosa-MG, v.34, n.5, p.825-833, 2010
Rejmánek, M. 1996. Species richness and resistance to invasions. Pp 153-172. In Orians, G.; Dirzo, R. e Cushman, J.H. (eds.) Biodiversity and ecosystem processes in tropical forests. New York: Springer.

Rejmánek, M. 1999. Invasive plant species and invasible ecosystems. Pp 79-102. In Sandlund, O.T.; Schei, P.J. \& Viken, A. (eds.) Invasive species and biodiversity management. Dordrecht, Netherlands: Kluwer.

Rejmánek, M. et al. 2002. Biological invasions: politics and the discontinuity of ecological terminology. Bulletin of the Ecological Society of America 83:131-133.

Resende, A.V. e Kondo, M.K. 2001. Leguminosas e recuperação de áreas degradadas. Informe Agropecuário 22(210):46-56.

Richardson, D.M., Pysek, P., Rejmánek, M., Barbour, M.G., Panetta, F.D. \& West, C.J. 2000. Naturalization and invasion of alien plants: concepts and definitions. Diversity and Distributions 6:93-107.

Scherer, L.M. et al. 2005. Allellopatic effects of aqueous extracts of leucena (Leucaena leucocephala Wit) leave and fruit on germination and root growth of canafístula (Peltophorum dubium Spreng). Semina: Ciências Biológicas e Saúde 26(2):161-166.

Schofield, E.K. 1989. Effects of introduced plants and animals on island vegetation: examples from the Galapagos Archipelago. Conservation Biology 3:227-238.

Seiffert, N.F.; Thiago, L.R.L.S. 1983. Legumineira: cultura forrageira para produção de proteína: guandu (Cajanus cajan). EMBRAPA-CNPGC, 52p. 1983. (Circular Técnica 13).

Silveira, E.R. e Durigan, G. 2004. Recuperação de matas ciliares: estrutura da floresta e regeneração natural aos dez anos em diferentes modelos de plantio na Fazenda Canaçu, Tarumã, SP. Pp. 325347. In: Vilas Boas, O. e Durigan, G. (Orgs.).

Pesquisas em conservação e recuperação ambiental no Oeste Paulista: resultados da cooperação Brasil/Japão. São Paulo: Páginas \& Letras. 
Siqueira, J.C.S. 2006. Bioinvasão vegetal: dispersão e propagação de espécies nativas e invasoras exóticas no campus da Pontifícia Universidade Católica do Rio de Janeiro (PUCRio). Pesquisas, Botânica 57:319-330.

Smith, C.W. 1985. Impact of alien plants on Hawai'i's native biota. Pp. 180-250. In: Stone, C.P. and J. e Scott, M. (eds.). Hawaii'i's Terrestrial Ecosystems: Preservation and Management. Cooperative National Park Resources Studies Unit, University of Hawaii, Manoa.

Socolowski, F. e Takaki, M. 2007. Germinação de sementes e emergência de plântulas de Tabebuia rosea (Bertoloni) A.P.DC.

(Bignoniaceae), uma espécie exótica com potencial invasor. Revista Árvore V.31, n.2, p. 229-238.

Staples, G.W.; Herbst, D. e Imada, C.T. 2000.

Survey of invasive or potentially invasive cultivated plants in Hawai'i. Bishop Museum Press. 35 p. (Bishop Museum Ocasional Papers n. 65).

Veloso, H.P.; Rangel Filho, A.L.; Lima, J.C.A. 1991. Classificação da vegetação brasileira, adaptada a um sistema universal. Rio de Janeiro: IBGE, Departamento de Recursos Naturais e Estudos Ambientais.
Vitousek, P. 1986. Biological invasions and ecosystem properties: can species make a difference? Pp 163-176 in H.A. Mooney \& J.A. Drake (eds.) Ecology of biological invasions of North America and Hawaii. Springer-Verlag, New York.

Wagner, W.L.; Herbst, D.R. e Sohmer, S.H. 1999. Manual of the flowering plants of Hawaii. Revised edition. Honolulu: University of Hawai'i Press/Bishop Museum Press. (Bernice P. Bishop Museum special publication).

Wilcove, D.S. et al. 1998. Quantifying threats to imperiled species in the United States.

Bioscience 48:607-615.

Williamson, M. \& Fitter, A. 1996. The varying success of invaders. Ecology 77:1661-1666.

Woitke, M. e Dietz, H. 2002. Shifts in dominance of native and invasive plants in experimental patches of vegetations. Perspectives in Plant Ecology, Evolution and Systematics 5(3):165-184.

Yamamoto, L.F. et al. 2007. Síndromes de polinização e de dispersão em fragmentos da Floresta Estacional Semidecídua Montana, SP, Brasil. Acta Botanica Brasilica 21(3):553-573.

Yoshida, K. e Oka, S. 2004. Invasion of Leucaena leucocephala and its effects on the native plant community in the Ogasawara (Bonin) Islands. Weed Technology 18:1371-1375. 